\title{
On the relationship between solutions of stochastic and random differential inclusions
}

\author{
T.CARABAllo ${ }^{1}$, J.A.LAngA ${ }^{1}$, J.VAlero ${ }^{2}$ \\ 1 Departamento de Ecuaciones Diferenciales y Análisis Numérico, \\ Universidad de Sevilla, \\ Apdo. de Correos 1160, 41080-Sevilla, Spain. \\ e-mails: caraball@cica.es ; langa@numer.us.es \\ 2 Universidad Cardenal Herrera CEU, \\ Comissari 3, 03203 Elche, Alicante. Spain. \\ e-mail: valer.el@ceu.es
}

\begin{abstract}
Some results on the relationship of the solutions of a stochastic differential inclusion and the corresponding random differential inclusion obtained after a change of variable are proved. As a consequence, we obtain the pullback convergence of the solutions of the stochastic inclusion to a compact random set. The cases of a reaction-diffusion inclusion perturbed by additive and multiplicative noises are considered.
\end{abstract}

\section{Introduction}

In our previous works Caraballo et al. [4, 5, 6], the concept of multivalued random dynamical system (MRDS) has been introduced and some applications to stochastic differential inclusions have been considered. In fact, a reaction-diffusion inclusion perturbed by additive and multiplicative noise is considered. As in the single-valued case, the construction of the MRDS relies on the possibility of performing a suitable change of variable driving the stochastic inclusion to a deterministic one depending on a random parameter (a random inclusion), so that the deterministic ideas can be properly adapted to this situation.

However, there exits a result due to Da Prato and Frankowska [7] which guarantees the existence of stochastic solutions for a more general noise than the ones considered in $[4,5,6]$.

In both situations, we do not have uniqueness of solutions. To define the MRDS in the first case, we take the union of all the solutions to the problem. Consequently, one can think about the relationship between the solutions of the stochastic inclusion and the random inclusion obtained after the change of variable. This is our main aim in this paper. Indeed, we shall prove that in our two particular situations (i.e., additive and multiplicative noises) each solution of the stochastic inclusion correspondes to a solution in the random one. The converse is true under some additional conditions on the solutions of the random inclusion.

It is shown in $[4,5,6]$ that the random inclusion generates a perfect cocycle having a compact global random attractor. As a consequence, all solutions of the stochastic inclusion with the initial condition in a bounded set of the phase space converge uniformly (in the sense of pullback attraction) to this attractor in the Hausdorff semidistance. This justifies the interest of the 
results of $[4,5,6]$, since they give some information on the asymptotic behaviour of the solutions of the stochastic inclusion.

Hopefully, it will be possible in the future to give some light on the possibility of considering more general situations than the ones treated up to date. As far as we know, this problem remains interesting even in the single-valued case (see also Capinski and Cutland [3] for another especial case of Navier-Stokes equation, and Imkeller and Schmalfuss [8] for a general change of variables relating stochastic and random differential equations).

\section{The additive case}

Let $H$ be a real separable Hilbert space with the norm $\|\cdot\|$ and the scalar product $\langle\cdot, \cdot\rangle$. The linear operator $A: D(A) \subset H \rightarrow H$ is called m-dissipative if

$$
<A(y), y>\leq 0 \text {, for all } y \in D(A)
$$

and $\operatorname{Im}(A-\lambda I)=H$, for any $\lambda>0$.

Let $A$ be a linear, m-dissipative, self-adjoint and densely defined operator (i.e. $\overline{D(A)}=H$ ) . It follows from these properties that $-A=\partial \varphi$ for some proper, lower semicontinuous convex function $\varphi: H \rightarrow(-\infty,+\infty]$ (see Barbu [2, p.60]).

Moreover, Philips-Lumer Theorem implies that $A$ is the infinitesimal generator of a semigroup of class $C_{0}$ denoted by $S(t)$. Since $A$ is m-dissipative, it is known that $\|S(t)\| \leq 1$.

The operator $S(t)$ is assumed to be compact for any $t>0$.

Consider the equation

$$
\left\{\begin{array}{l}
\frac{d u(t)}{d t}=A u(t)+f(t), 0 \leq t \leq T \\
u(0)=u_{0} \in H
\end{array}\right.
$$

The function $u:[0, T] \rightarrow H$ is called a strong solution to (1) if $u(\cdot) \in C([0, T], H), u(0)=$ $u_{0}, u$ is absolutely continuous on compact sets of $(0, T), u$ is a.e. differentiable in $(0, T)$, and $\frac{d u(t)}{d t}=A u(t)+f(t)$ for a.a. $t \in(0 . T)$.

It is well known that for any $f(\cdot) \in L^{2}(0, T ; H)$ the equation has a unique strong solution (see Barbu [2, p.189]). Moreover, it is well known that

$$
u(t)=S(t) u_{0}+\int_{0}^{t} S(t-s) f(s) d s, \text { for any } t \geq 0 .
$$

Consider the stochastic differential inclusion (see Caraballo et al. [4, 6] for more details)

$$
\left\{\begin{array}{l}
\frac{d u(t)}{d t} \in A u(t)+F(u(t))+\sum_{i=1}^{m} \phi_{i} \frac{d w_{i}(t)}{d t}, 0 \leq t \leq T \\
u(0)=u_{0} \in H
\end{array}\right.
$$

where $F: H \rightarrow 2^{H}$ satisfies:

(F1) $F$ has closed, bounded, convex, non-empty values. 
(F2) There exists $C>0$ such that

$$
\operatorname{dist}_{H}(F(u), F(v)) \leq C\|u-v\|, \forall u, v \in H
$$

where ' dist $_{H}$ ' denotes the Hausdorff metric.

Let $\zeta(t)=\sum_{i=1}^{m} \phi_{i} w_{i}(t)$. Let us consider the Wiener probability space $(\Omega, \mathcal{F}, \mathbb{P})$ defined by

$$
\Omega=\left\{\omega=\left(w_{1}(\cdot), \ldots, w_{m}(\cdot)\right) \in C\left(\mathbb{R}, \mathbb{R}^{m}\right) \mid \omega(0)=0\right\},
$$

equipped with the Borel $\sigma$-algebra $\mathcal{F}$, the Wiener measure $\mathbb{P}$, and the usual uniform convergence on bounded sets of $\mathbb{R}$. Each $\omega \in \Omega$ generates a map $\zeta(\cdot)=\sum_{i=1}^{m} \phi_{i} w_{i}(\cdot) \in C(\mathbb{R}, H)$ such that $\zeta(0)=0$.

Consider the maps $\rho_{s, t}: \Omega \rightarrow \Omega$

$$
\rho_{s, t}(\omega)(\tau)=\left\{\begin{array}{c}
\omega(s), \text { si } \tau \leq s \\
\omega(\tau), \text { si } s \leq \tau \leq t \\
\omega(t), \text { si } \tau \geq t
\end{array}\right.
$$

and define the $\sigma$-algebras $\mathcal{F}_{s, t}=\rho_{s, t}^{-1} \mathcal{F}$. It is clear that $\mathcal{F}_{s, t} \subset \mathcal{F}_{s^{\prime}, t^{\prime}} \subset \mathcal{F}, \forall s^{\prime} \leq s \leq t \leq t^{\prime}$.

A process $u(t, \omega):[0, \infty) \times \Omega \rightarrow H$ is said to be adapted if $u(t, \cdot)$ is $\mathcal{F}_{0, t}$ measurable for any $t \geq 0$.

Now, Theorem 2.1 from [7] provides the existence of at least one solution $u\left(\cdot, \omega, u_{0}\right)$ to $(2)$ for any $u_{0} \in H$, that is, an adapted process with values in $H$ such that:

1. $u\left(\cdot, \omega, u_{0}\right)$ is continuous for $\mathbb{P}$-a.a. $\omega \in \Omega$.

2. $u\left(0, \omega, u_{0}\right)=u_{0}$.

3. For any $t \in[0, T]$

$$
u(t)=S(t) u_{0}+\int_{0}^{t} S(t-s) f(s) d s+\sum_{i=1}^{m} \int_{0}^{t} S(t-s) \phi_{i} d w_{i}(s),
$$

being $f(s, \omega)$ an adapted process such that

$$
\begin{gathered}
f(s, \omega) \in F(u(s, \omega)), \text { for a.a. }(s, \omega) \in(0, T) \times \Omega, \\
\mathbb{E}\left(\int_{0}^{T}\|f(s)\|^{2} d s\right)<\infty .
\end{gathered}
$$

Note that, since $T>0$ is arbitrary, we can extend any solution to the whole $[0,+\infty)$.

Consider now the change of variable $v(t)=u(t)-\zeta(t)$. Then, inclusion (2) becomes (formally)

$$
\left\{\begin{array}{l}
\frac{d v(t)}{d t} \in A v(t)+F(v(t)+\zeta(t))+A \zeta(t), 0 \leq t \leq T, \\
v(0)=u_{0} .
\end{array}\right.
$$

For any $u_{0} \in H$ and $\omega \in \Omega$ there exists a strong solution $v\left(\cdot, \omega, u_{0}\right)$ to (3), i.e., a strong solution to (1) for some $f(\cdot) \in L^{2}(0, T ; H)$ such that $f(t) \in F(v(t)+\zeta(t))+A \zeta(t)$, a.e. in $(0, T)$.

Let us denote the solutions to (2) and (3) by $u(\cdot)=I_{s}\left(u_{0}\right) f(\cdot)$ and $v(\cdot)=I_{r}\left(u_{0}\right) f(\cdot)$, respectively. Then, we can prove the following results. 
Proposition 1 If $u(\cdot)=I_{s}\left(u_{0}\right) f(\cdot)$, then the function defined as $v(\cdot)=u(\cdot)-\zeta(\cdot)$ satisfies $v(\cdot)=I_{r}\left(u_{0}\right)(f(\cdot)+A \zeta(\cdot))$. In other words, if $u(\cdot)$ is a solution of the stochastic inclusion (2) in the sense of Da Prato and Frankowska, and we define the function $v(\cdot)$ by the equality $v(\cdot)=u(\cdot)-\zeta(\cdot)$, then $v(\cdot)$ is a strong solution to (2) but substituting the original $f(t)$ in the right-hand side of (1) by $f(t)+A \zeta(t)$.

Proof. Since $u(\cdot)$ is a solution to (2) we have

$$
u(t)=S(t) u_{0}+\int_{0}^{t} S(t-s) f(s) d s+\sum_{i=1}^{m} \int_{0}^{t} S(t-s) \phi_{i} d w_{i}(s) .
$$

Let $Y(s)=\sum_{i=1}^{m} S(t-s) \phi_{i} w_{i}(s)$. Since $d S(t) u_{0}=A S(t) u_{0} d t$, we easily get

$$
d Y(s)=S(t-s) \sum_{i=1}^{m} \phi_{i} d w_{i}(s)-A S(t-s) \sum_{i=1}^{m} \phi_{i} w_{i}(s) d s .
$$

Hence, using the fact that $A$ and $S(t)$ commute, we have

$$
\sum_{i=1}^{m} \phi_{i} w_{i}(t)=\sum_{i=1}^{m} \int_{0}^{t} S(t-s) \phi_{i} d w_{i}(s)-\sum_{i=1}^{m} \int_{0}^{t} S(t-s) A \phi_{i} w_{i}(s) d s .
$$

Now, combining (4) and (5), we obtain

$$
v(t)=u(t)-\zeta(t)=S(t) u_{0}+\int_{0}^{t} S(t-s)(f(s)+A \zeta(s)) d s .
$$

It follows $v(\cdot)=I_{r}\left(u_{0}\right)(f(\cdot)+A \zeta(\cdot))$.

Proposition 2 If $v(\cdot)=I_{r}\left(u_{0}\right) f(\cdot)$, being $f(t)$ an adapted process such that $\mathbb{E}\left(\int_{0}^{T}\|f(s)\|^{2} d s\right)<$ $\infty$, then $u(\cdot):=v(\cdot)+\zeta(\cdot)=I_{s}\left(u_{0}\right)(f(\cdot)-A \zeta(\cdot))$.

Proof. Since $v(\cdot)$ is a strong solution of (1), we have

$$
\begin{aligned}
v(t) & =S(t) u_{0}+\int_{0}^{t} S(t-s) f(s) d s \\
& =S(t) u_{0}+\int_{0}^{t} S(t-s) g(s) d s+\sum_{i=1}^{m} \int_{0}^{t} S(t-s) A \phi_{i} w_{i}(s) d s
\end{aligned}
$$

where $g(s)=f(s)-A \zeta(s) \in F(v(s)+\zeta(s))$, a.e. on $(0, T)$.

Using (5) we obtain

$$
u(t)=v(t)+\zeta(t)=S(t) u_{0}+\int_{0}^{t} S(t-s) g(s) d s+\sum_{i=1}^{m} \int_{0}^{t} S(t-s) \phi_{i} d w_{i}(s) .
$$

Hence, $u(\cdot)=I_{s}\left(u_{0}\right) g(\cdot)$. (Observe that $u(\cdot)$ is adapted since $f$ and $\zeta$ also are.)

Now, notice that the random differential inclusion (3) generates a perfect mutivalued cocycle $G: \mathbb{R}_{+} \times \Omega \times H \rightarrow C(H)$ by means of

$$
G(t, \omega) u_{0}=\bigcup_{v(\cdot) \in \mathcal{D}\left(u_{0}, \omega\right)}\{v(t)+\zeta(t)\},
$$


where

$$
\mathcal{D}\left(u_{0}, \omega\right)=\{v(\cdot): v(\cdot) \text { is a strong solution to }(3)\} .
$$

Moreover, $G$ has compact values (see Caraballo et al. [4]).

Definition 3 The closed random set $\omega \mapsto \mathcal{A}(\omega)$ (that is, a measurable map with closed values) is called a global random attractor of $G$ if:

i) $G(t, \omega) \mathcal{A}(\omega)=\mathcal{A}\left(\theta_{t} \omega\right)$, for all $t \geq 0, \mathbb{P}-$ a.s (that is, it is strictly invariant);

ii) For all bounded $D \subset X$,

$$
\lim _{t \rightarrow+\infty} \operatorname{dist}\left(G\left(t, \theta_{-t} \omega\right) D, \mathcal{A}(\omega)\right)=0, \mathbb{P}-a . s .
$$

iii) $\mathcal{A}(\omega)$ is compact $\mathbb{P}-$ a.s.

Property ii) means that the initial moment of time goes to $-\infty$ and the final moment is 0 . This is called the pullback convergence in the literature (Kloeden and Schmalfuss [9]) .

If we assume the following conditions:

(H1) There exist constants $\delta>0, M \geq 0$ such that

$$
\langle y, u\rangle \leq(-\delta+\varepsilon)\|u\|^{2}+M, \text { for all } u \in D(A), y \in F(u),
$$

where $\varepsilon \geq 0$ is the biggest constant such that

$$
\langle A u, u\rangle \leq-\varepsilon\|u\|^{2} .
$$

(H2) The level sets

$$
M_{R}=\{u \in D(\varphi) \mid\|u\| \leq R, \varphi(u) \leq R\}
$$

are compact in $H$ for any $R>0$.

Then, it is proved in $[4$, Theorem 16] that $G$ has the global random attractor $\mathcal{A}(\omega)$, which is measurable with respect to the $\sigma$-algebra $\mathcal{F}$.

As a consequence of Proposition 1 we have $u\left(t, \omega, u_{0}\right) \in G(t, \omega) u_{0}$, for all $\left(t, u_{0}\right) \in \mathbb{R}_{+} \times$ $H, \omega \in \Omega$ and $u\left(\cdot, \omega, u_{0}\right) \in \mathcal{L}\left(u_{0}, \omega\right)$, where

$$
\mathcal{L}\left(u_{0}, \omega\right)=\left\{u\left(\cdot, \omega, u_{0}\right): u\left(\cdot, \omega, u_{0}\right) \text { is a solution to }(2)\right\} .
$$

We then obtain that all the solutions of inclusion (2) with initial conditions on a bounded set converge uniformly to $\mathcal{A}(\omega)$ in the sense of Definition 3 .

Corollary 4 Assuming $(H 1)-(H 2)$, for any bounded set $B$ it holds

$$
\lim _{t \rightarrow+\infty} \sup _{u \in \mathcal{L}\left(u_{0}, \omega\right)} \sup _{u_{0} \in B} \operatorname{dist}\left(u\left(t, \theta_{-t} \omega, u_{0}\right), \mathcal{A}(\omega)\right)=0 .
$$


These results can be applied to the following reaction-diffusion inclusion

$$
\left\{\begin{array}{c}
\frac{\partial u}{\partial t} \in \Delta u+f(u)+h+\sum_{i=1}^{m} \phi_{i} \frac{d w_{i}(t)}{d t}, \text { on } \mathcal{O} \times(0, T), \\
u=0, \text { on } \partial \mathcal{O} \times(0, T), \\
u(x, 0)=u_{0}(x) \text { on } \mathcal{O},
\end{array}\right.
$$

where $\mathcal{O} \subset \mathbb{R}^{n}$ is an open bounded subset with smooth boundary $\partial \mathcal{O}, h(\cdot) \in L_{2}(\mathcal{O})$ and $f: \mathbb{R} \rightarrow$ $2^{\mathbb{R}}$ is a multivalued map with non-empty, compact convex values. Assume that $f$ is Lipschitz, i.e. there exists $C \geq 0$ such that

$$
\operatorname{dist}_{H}(f(x), f(z)) \leq C\|x-z\|, \text { for all } x, z \in \mathbb{R} .
$$

Define the operators $A: D(A) \rightarrow H, F: H \rightarrow 2^{H}, H=L_{2}(\mathcal{O})$,

$$
\begin{gathered}
A u=\Delta u, \\
F(u)=\{y \in H: y(x) \in f(u(x))+h(x), \text { a.e. on } \mathcal{O}\},
\end{gathered}
$$

with $D(A)=H^{2}(\mathcal{O}) \cap H_{0}^{1}(\mathcal{O})$. It is assumed that $\phi_{i} \in D(A)$. The map $-A$ is the subdifferential of a proper, convex, lower semicontinuous function $\varphi$ and the map $F$ satisfies $(F 1)-(F 2)$. Moreover, condition (H2) is satisfied and $\overline{D(\varphi)}=H$ (see Melnik and Valero [10, Section 3.2.2.]). On the other hand, it is well known that the operator $A$ satisfies all the conditions assumed before.

Hence, Propositions 1-2 hold.

If we also assume the existence of $M \geq 0, \delta>0$ such that

$$
z s \leq\left(\lambda_{1}-2 \delta\right)|s|^{2}+M_{1}, \text { for all } s \in \mathbb{R}, z \in f(s),
$$

where $\lambda_{1}$ is the first eigenvalue of $-\Delta$ in $H_{0}^{1}(\Omega)$, then $(H 1)$ is also satisfied (see [4, Theorem 17]). It follows that Corollary 4 holds.

\section{The multiplicative case}

Let us now consider the multiplicative case from Caraballo et al. [5]. That is, let us consider the following stochastic differential inclusion in the Stratonovich sense

$$
\left\{\begin{array}{l}
\frac{d u(t)}{d t} \in A u(t)+F(u(t))+\sigma u(t) \circ \frac{d w(t)}{d t}, 0 \leq t \leq T, \\
u(0)=u_{0} \in H,
\end{array}\right.
$$

where $\sigma \in \mathbb{R}, F: H \rightarrow 2^{H}$ satisfies $(F 1)-(F 2)$ and now the Wiener probability space $(\Omega, \mathcal{F}, \mathbb{P})$ is defined by

$$
\Omega=\{\omega=w(\cdot) \in C(\mathbb{R}, \mathbb{R}) \mid \omega(0)=0\},
$$

equipped with the Borel $\sigma$-algebra $\mathcal{F}$, the Wiener measure $\mathbb{P}$, and the usual uniform convergence on bounded sets of $\mathbb{R}$.

The change of variable which takes the stochastic inclusion (10) into a random one is different from the one in the previous section. Indeed, set $\alpha(t)=\alpha(t, \omega)=\mathrm{e}^{-\sigma w_{t}(\omega)}$. Using the change $v(t)=\alpha(t) u(t)$, inclusion (10) becomes (formally)

$$
\left\{\begin{array}{l}
\frac{d v(t)}{d t} \in A v(t)+\alpha(t) F\left(\alpha^{-1}(t) v(t)\right), 0 \leq t \leq T, \\
v(0)=u_{0} \in H
\end{array}\right.
$$


Thus, as in Section 2 and taking into account the relation between the Ito and Stratonovich integral in this special case, Theorem 2.1 in [7] also ensures the existence of at least one solution to (10) satisfying:

1. $u\left(\cdot, \omega, u_{0}\right)$ is continuous for $\mathbb{P}$-a.a. $\omega \in \Omega$.

2. $u\left(0, \omega, u_{0}\right)=u_{0}$.

3. For any $t \in[0, T]$

$$
u(t)=S(t) u_{0}+\int_{0}^{t} S(t-s) f(s) d s+\int_{0}^{t} S(t-s) u(s) \circ d w(s)
$$

where $f(s, \omega)$ is an adapted process such that

$$
\begin{gathered}
f(s, \omega) \in F(u(s, \omega)), \text { for a.a. }(s, \omega) \in(0, T) \times \Omega, \\
\mathbb{E}\left(\int_{0}^{T}\|f(s)\|^{2} d s\right)<\infty .
\end{gathered}
$$

On the other hand, given $u_{0} \in H$ and $\omega \in \Omega$, there exists a strong solution of (11), i.e. a strong solution of (1) for some $f(\cdot) \in L^{2}(0, T ; H)$ such that $f(t) \in \alpha(t) F\left(\alpha^{-1}(t) v(t)\right)$ a.e. in $(0, T)$.

Using again a similar notation to that one in Section 2, i.e. $u(\cdot)=J_{s}\left(u_{0}\right) f(\cdot)$ for the solutions of $(10)$, and $v(\cdot)=J_{r}\left(u_{0}\right) f(\cdot)$ for the solutions of $(11)$, we can prove the following results.

Proposition 5 If $u(\cdot)=J_{s}\left(u_{0}\right) f(\cdot)$, then $v(\cdot)=\alpha(\cdot) u(\cdot)=J_{r}\left(u_{0}\right)(\alpha(\cdot) f(\cdot))$. In other words, if $u(\cdot)$ is a solution to the stochastic inclusion (10) in the sense of Da Prato and Frankowska, and we define the function $v(\cdot)$ by $v(\cdot)=\alpha(\cdot) u(\cdot)$, then $v(\cdot)$ is a strong solution to (11) but substituting the original $f(t)$ in the right-hand side of (1) by $\alpha(t) f(t)$.

Proof. Let $u(\cdot)$ be a solution to (10). Then, we have

$$
u(t)=S(t) u_{0}+\int_{0}^{t} S(t-s) f(s) d s+\sigma \int_{0}^{t} S(t-s) u(s) \circ d w(s),
$$

where $f(s) \in F(u(s))$ a.e. in $(0, T) \times \Omega$.

Let $Y(s)=\alpha(s) S(t-s) u(s), 0 \leq s \leq t$. Then, it follows

$$
d Y(s)=-\sigma \alpha(s) S(t-s) u(s) \circ d w(s)+\alpha(s) d[S(t-s) u(s)],
$$

and, by a direct integration

$$
\begin{aligned}
Y(t)-Y(0)=- & \sigma \int_{0}^{t} \alpha(s) S(t-s) u(s) \circ d w(s) \\
& +\int_{0}^{t} \alpha(s) d[S(t-s) u(s)] .
\end{aligned}
$$

Now, we work with the last term in (13). 
Observe that

$$
\begin{aligned}
& d[S(t-s) u(s)] \\
& =d\left[S(t-s)\left\{S(s) u_{0}+\int_{0}^{s} S(s-\tau) f(\tau) d \tau+\sigma \int_{0}^{s} S(s-\tau) u(\tau) \circ d w(\tau)\right\}\right] \\
& =d\left[S(t) u_{0}+\int_{0}^{s} S(t-\tau) f(\tau) d \tau+\sigma \int_{0}^{s} S(t-\tau) u(\tau) d w(\tau)\right] \\
& =S(t-s) f(s) d s+\sigma S(t-s) u(s) \circ d w(s),
\end{aligned}
$$

and, consequently, (13) turns into

$$
\begin{aligned}
Y(t)-Y(0) & =-\sigma \int_{0}^{t} \alpha(s) S(t-s) u(s) \circ d w(s) \\
& +\int_{0}^{t} S(t-s) \alpha(s) f(s) d s \\
& +\int_{0}^{t} \sigma \alpha(s) S(t-s) u(s) \circ d w(s) \\
& =\int_{0}^{t} S(t-s) \alpha(s) f(s) d s .
\end{aligned}
$$

Taking into account that $Y(t)=v(t)$ and $Y(0)=S(t) u_{0}$, it holds

$$
v(t)=S(t) u_{0}+\int_{0}^{t} S(t-s) \tilde{f}(s) d s
$$

where $\tilde{f}(s)=\alpha(s) f(s)$.

Proposition 6 If $v(\cdot)=J_{r}\left(u_{0}\right) f(\cdot)$, being $f(t)$ an adapted process such that $\mathbb{E}\left(\int_{0}^{T}\|f(s)\|^{2} d s\right)<$ $\infty$, then $u(\cdot)=\alpha^{-1}(\cdot) v(\cdot)=J_{s}\left(u_{0}\right)(\tilde{f}(\cdot))$, where $\tilde{f}(s)=\alpha^{-1}(s) f(s)$.

Proof. Since $v(\cdot)$ is a strong solution to (11), we have

$$
v(t)=S(t) u_{0}+\int_{0}^{t} S(t-s) f(s) d s,
$$

where $f(s) \in \alpha(s) F\left(\alpha^{-1}(s) v(s)\right)$, a.e. on $(0, T)$.

Let us denote $Y(s)=\alpha^{-1}(s) S(t-s) v(s)=\mathrm{e}^{\sigma w_{s}(\omega)} S(t-s) v(s), 0 \leq s \leq t$. Then, arguing as in the preceding proof, it follows

$$
\begin{aligned}
d Y(s) & =\sigma \mathrm{e}^{\sigma w_{s}} S(t-s) v(s) \circ d w_{s}+\mathrm{e}^{\sigma w_{s}} d[S(t-s) v(s)] \\
& =\sigma \mathrm{e}^{\sigma w_{s}} S(t-s) v(s) \circ d w_{s}+\mathrm{e}^{\sigma w_{s}} S(t-s) f(s) d s,
\end{aligned}
$$

and, after integration, it yields

$$
\begin{aligned}
Y(t)-Y(0) & =\int_{0}^{t} S(t-s) \alpha^{-1}(s) f(s) d s \\
& +\sigma \int_{0}^{t} S(t-s) \alpha^{-1}(s) v(s) \circ d w_{s} .
\end{aligned}
$$


Setting $u(t)=\alpha^{-1}(t) v(t), \tilde{f}(t)=\alpha^{-1}(t) f(t)$, it holds

$$
u(t)=S(t) u_{0}+\int_{0}^{t} S(t-s) \tilde{f}(s) d s+\sigma \int_{0}^{t} S(t-s) u(s) \circ d w_{s} .
$$

Therefore the proof is complete since the adaptedness of $u$ is immediate.

Now, as in the additive case, differential inclusion (11) generates a perfect mutivalued cocycle $G_{M}: \mathbb{R}_{+} \times \Omega \times H \rightarrow C(H)$ by setting

$$
G_{M}(t, \omega) u_{0}=\bigcup_{v(\cdot) \in \mathcal{D}\left(u_{0}, \omega\right)} \alpha^{-1}(t) v(t),
$$

where

$$
\mathcal{D}\left(u_{0}, \omega\right)=\{v(\cdot): v(\cdot) \text { is a solution to }(11)\} .
$$

Moreover, $G_{M}$ has compact values (see Caraballo et al. [5] for more details).

If we assume $(H 1)-(H 2)$, then it is proved in [5, Theorem 26] that $G$ has the global random attractor $\mathcal{A}(\omega)$, which is measurable with respect to the $\sigma$-algebra $\mathcal{F}$.

As a consequence of Proposition 5 we have $u\left(t, \omega, u_{0}\right) \in G_{M}(t, \omega) u_{0}$, for all $\left(t, u_{0}\right) \in \mathbb{R}_{+} \times$ $H, \omega \in \Omega$ and $u\left(\cdot, \omega, u_{0}\right) \in \mathcal{L}_{M}\left(u_{0}, \omega\right)$, where

$$
\mathcal{L}_{M}\left(u_{0}, \omega\right)=\left\{u\left(\cdot, \omega, u_{0}\right): u\left(\cdot, \omega, u_{0}\right) \text { is a solution to }(10)\right\} .
$$

We obtain then that all the solutions to inclusion (10) with initial conditions on a bounded set converge uniformly to $\mathcal{A}(\omega)$ in the sense of Definition 3.

Corollary 7 Assuming $(H 1)-(H 2)$, for any bounded set $B$ it holds

$$
\lim _{t \rightarrow+\infty} \sup _{u \in \mathcal{L}_{M}\left(u_{0}, \omega\right)} \sup _{u_{0} \in B} \operatorname{dist}\left(u\left(t, \theta_{-t} \omega, u_{0}\right), \mathcal{A}(\omega)\right)=0
$$

These results can be applied to the following reaction-diffusion inclusion

$$
\left\{\begin{array}{c}
\frac{\partial u}{\partial t} \in \Delta u+f(u)+h+\sigma u(t) \circ \frac{d w(t)}{d t}, \text { on } \mathcal{O} \times(0, T), \\
u=0, \text { on } \partial \mathcal{O} \times(0, T), \\
u(x, 0)=u_{0}(x) \text { on } \mathcal{O},
\end{array}\right.
$$

where $\mathcal{O} \subset \mathbb{R}^{n}, h, f, \mathrm{~F}$ and $A$ are defined as for (7).

Hence, Propositions 5-6 hold.

If we assume also the existence of $M \geq 0, \delta>0$ such that

$$
z s \leq\left(\lambda_{1}-2 \delta\right)|s|^{2}+M_{1}, \text { for all } s \in \mathbb{R}, z \in f(s),
$$

where $\lambda_{1}$ is the first eigenvalue of $-\Delta$ in $H_{0}^{1}(\Omega)$, then $(H 1)$ is also satisfied (see [5, Theorem 27]). It follows that Corollary 7 holds. 


\section{Conclusions}

In our opinion, the results obtained in Corollaries 4, 7 justify the construction of the multivalued random dynamical systems we have done in some previous papers.

It would be fine to construct a cocycle from the solutions of the stochastic inclusion in a similar manner as we have done for the random inclusion and prove the existence of a global

attractor in this case. Such a result could give the key to study a stochastic inclusion containing a more general noise (that is, different from the additive and multiplicative cases). Note that the problem of considering more general noises is open also for stochastic equations, an some improvementes have been done in [8] and [3].

It would be also of interest to study whether this cocycle would coincide or not with the cocycle generated by the random inclusion after the change of variable.

\section{References}

[1] J.P. Aubin And H. Frankowska, Set-Valued Analysis, Birkhäuser, Boston (1990).

[2] V. BARBu, Nonlinear Semigroups and Differential Equations in Banach Spaces, Editura Academiei, Bucuresti (1976).

[3] M. Capinski and N.J. Cutland, Existence of global stochastic flow and attractors for Navier-Stokes equations, Prob. Th. and Rel. Fields 115(1999), 121-151.

[4] T. Caraballo, J.A. Langa and J. Valero, Global attractors for multivalued random dynamical systems, Nonlinear Analysis TMA, to appear.

[5] T. Caraballo, J.A. Langa and J. Valero, Global attractors for multivalued random dynamical systems generated by random differential inclusions with multiplicative noise, $J$. Math. Anal. Appl., to appear.

[6] T. Caraballo, J.A. Langa and J. Valero, Global attractors for multivalued random semiflows generated by random differential inclusions with additive noise, C.R. Acad. Sci. Paris, t. 332, Série I (2001), 131-136.

[7] G. Da Prato and H. Frankowska, A stochastic Filippov theorem, Stochastic Anal. Appl., 38 (5)(1994), 409-426.

[8] P. Imkeller, B. Schamalfuss, The conjugacy of stochastic and random differential equations and the existence of global attractors, preprint.

[9] P.E. Kloeden \& B. Schmalfuss, Cocyle attractors of variable time step discretizations of Lorenzian systems. J. Difference Eqns. Applns. 3 (1997) pp. 125-145.

[10] V.S. Melnik and J. Valero, On Attractors of multivalued semi-flows and differential inclusions, Set-Valued Anal. 6 (1998), 83-111.

[11] A.A. Tolstonogov, On solutions of evolution inclusions.I, Sibirsk. Mat. Zh. 33, 3 (1992), 161-174 (English translation in Siberian Math. J., 33, 3 (1992)). 